\title{
ADIABATIC INVARIANTS FOR STRINGS, MEMBRANES, AND BEAMS OF SLOWLY-VARYING DIMENSIONS*
}

BY

W. A. DAY

Hertford College, Oxford

1. Introduction. This paper is concerned with four problems which arise in elementary linear theories of the transverse vibration of strings, membranes, and beams. The problems are:
A. $T(t) u_{x x}=u_{t t} \quad$ and $\quad u(0, t)=u(L(t), t)=0$
B. $\left(x u_{x}\right)_{x}=u_{t t}$ and $u(L(t), t)=0$;
C. $T(t)\left(x u_{x}\right)_{x}=x u_{t t}$ and $u(L(t), t)=0$;
D. $u_{x x x x}+u_{t t}=0 \quad$ and $\quad u(0, t)=u_{x}(0, t)=u(L(t), t)=u_{x}(L(t), t)=0$.

In $\mathrm{A}$, it is envisaged that a string, of unit density, is stretched to a tension $T(t)$ and the end $x=0$ is held fixed. A heavy ring is moved along the string, its position being $x=L(t)$, and the effect of the ring is to nullify the displacement $u$.

In $\mathrm{B}$, a string (or chain) of unit density is suspended under gravity, and the gravitational acceleration is taken to be unity. The lower end $x=0$ is free and the tension is $x$, the $x$-axis being directly vertically upward. As in problem A, a heavy ring is moved along the string; the position of the ring is $x=L(t)$, and the ring nullifies the displacement.

In $\mathrm{C}, x$ is distance from the origin in the plane and would more usually be denoted by $r$. A membrane, of unit density, is stretched to a tension $T(t)$ and occupies the disk $0 \leqslant x \leqslant L(t)$. The displacement is assumed to depend on $x$ and $t$ only and to vanish at the moving boundary $x=L(t)$.

In $\mathrm{D}$, a beam, of unit density and unit flexural rigidity, undergoes transverse vibration and the end $x=0$ is clamped. A sleeve is moved along the beam, its position being $x=L(t)$, and the action of the sleeve is to nullify both the displacement $u$ and the slope $u_{x}$.

The behavior of $u$ is made complicated, in all four problems, by the fact that the interval $0 \leqslant x \leqslant L(t)$, to which the spatial variable $x$ belongs, varies with the time $t$. In cases $\mathrm{A}$ and $\mathrm{C}$, there is the additional complication of a coefficient $T(t)$ that varies with $t$.

${ }^{*}$ Received June 24, 1986.

(C) 1987 Brown University 
Both $L$ and $T$ are understood to be strictly positive and slowly-varying; what the latter qualification means will be explained later. Our methods would allow us to treat the, admittedly artificial, generalization of $\mathrm{B}$ in which the gravitational acceleration varies slowly with $t$.

It is supposed throughout that $u(x, t)$ is defined whenever $0 \leqslant x \leqslant L(t)$ and $0 \leqslant t<\infty$ and has as many continuous derivatives as our arguments require.

Our purpose is to study the behavior of the energies $E$ associated with the four problems. To within irrelevant factors, these are, respectively:

$$
\begin{aligned}
& \int_{0}^{I}\left(T u_{x}^{2}+u_{t}^{2}\right) d x, \\
& \int_{0}^{l}\left(x u_{x}^{2}+u_{t}^{2}\right) d x, \\
& \int_{0}^{L} x\left(T u_{x}^{2}+u_{t}^{2}\right) d x, \\
& \int_{0}^{L}\left(u_{x x}^{2}+u_{t}^{2}\right) d x .
\end{aligned}
$$

If $L$ (and $T$ as well in problems $A$ and C) were constant the same would be true of $E$, and so it is natural to ask how $E$ changes when $L$ and $T$ are slowly-varying. Two pieces of evidence suggest that it may be possible to obtain quantitative information about the behavior of $E$.

The first piece of evidence is provided by what is known about the Lorentz pendulum - a pendulum whose length is slowly-varying. In that case, the energy is approximately proportional to the frequency or, what amounts to the same thing, the product

$$
\text { (length) })^{1 / 2} \times \text { energy }
$$

is an adiabatic invariant.

Although there is an extensive literature on Lorentz's problem, we shall refer only to Littlewood's paper [1] (reprinted in the collection [2], pp. 356-365). At first sight, Littlewood's methods appear to be highly specific to Lorentz's problem but, and this is the second piece of evidence, they can be adapted to construct adiabatic invariants for certain partial differential equations with slowly-varying coefficients [3]. The methods of [3] show that for problems $\mathrm{A}$ and $\mathrm{C}$ the product $T^{-1 / 2} \cdot E$ is an adiabatic invariant provided $L$ is constant, but the possibility that $L$ might change was not contemplated in that paper.

What will be established here is that the products

$$
L \cdot T^{-1 / 2} \cdot E, \quad L^{1 / 2} \cdot E, \quad L \cdot T^{-1 / 2} \cdot E, \quad L^{2} \cdot E,
$$

are adiabatic invariants for problems $\mathrm{A}, \mathrm{B}, \mathrm{C}, \mathrm{D}$, respectively.

Problem B corresponds to a flexible pendulum of slowly-varying length $L$, and it is, therefore, not altogether surprising that the adiabatic invariant $L^{1 / 2} \cdot E$ should prove to be the same as for the Lorentz pendulum.

2. Adiabatic invariants for $\mathbf{A}, \mathbf{B}, \mathbf{C}$; some preliminary results. Problems $\mathrm{A}, \mathrm{B}, \mathrm{C}$ can be handled simultaneously by considering the differential equation

$$
T(t)\left(x^{a} u_{x}\right)_{x}=x^{b} u_{t t},
$$


in which the exponents $a$ and $b$ are nonnegative and $a<2+b$. (In $\mathrm{A}$, the exponents are $a=b=0$; in $\mathrm{B}$, the coefficient $T=1$ and the exponents are $a=1$ and $b=0$; in $\mathrm{C}$, the exponents are $a=b=1$.)

It is supposed that $u$ vanishes at the moving boundary $x=L$, i.e.,

$$
u(L(t), t)=0, \quad 0 \leqslant t<\infty .
$$

The additional condition

$$
u(0, t)=0, \quad 0 \leqslant t<\infty,
$$

is imposed when $a=0$ but not otherwise.

Our objective is to show that

$$
L^{(2+b-a) / 2} \cdot T^{-1 / 2} \cdot E
$$

is an adiabatic invariant; if so, $E$ must decrease as $L$ increases, because $a<2+b$, but must increase as $T$ increases. In order to verify our assertion we start by assembling a number of preliminary formulae and estimates.

First, we express the energy

$$
E=\int_{0}^{l}\left(T x^{a} u_{x}^{2}+x^{h} u_{t}^{2}\right) d x
$$

as the sum of the potential and kinetic energies

$$
J=\int_{0}^{I} T x^{a} u_{x}^{2} d x, \quad K=\int_{0}^{l} x^{b} u_{t}^{2} d x,
$$

and introduce the integrals

$$
F=\int_{0}^{L} x^{b} u^{2} d x, \quad G=2 \int_{0}^{L} x^{1+b} u_{x} u_{t} d x .
$$

The derivative of $E$ is

$$
\begin{aligned}
\dot{E} & =\left.\dot{L}\left(T x^{a} u_{x}^{2}+x^{b} u_{t}^{2}\right)\right|_{x=L}+\int_{0}^{L}\left(\dot{T} x^{a} u_{x}^{2}+2 T x^{a} u_{x} u_{x t}+2 x^{b} u_{t} u_{t t}\right) d x \\
& =\left.\dot{L}\left(T x^{a} u_{x}^{2}+x^{b} u_{t}^{2}\right)\right|_{x=L}+\dot{T} T^{-1} J+2 \int_{0}^{L}\left(T x^{a} u_{x} u_{x t}+x^{b} u_{t} u_{t t}\right) d x .
\end{aligned}
$$

According to (2.1),

$$
\begin{aligned}
\int_{0}^{l}\left(T x^{a} u_{x} u_{x t}+x^{b} u_{t} u_{t t}\right) d x & =\int_{0}^{l}\left(T x^{a} u_{x} u_{x t}+T u_{t}\left(x^{a} u_{x}\right)_{x}\right) d x \\
& =T \int_{0}^{l}\left(x^{a} u_{x} u_{t}\right)_{x} d x=\left.T\left(x^{a} u_{x} u_{t}\right)\right|_{x=L},
\end{aligned}
$$

where we have used the fact that $x^{a} u_{x} u_{t}$ vanishes at $x=0$ (when $a=0$ the additional boundary condition (2.3) is needed). Hence

$$
\dot{E}=\left.\left(\dot{L} T x^{a} u_{x}^{2}+\dot{L} x^{b} u_{t}^{2}+2 T x^{a} u_{x} u_{t}\right)\right|_{x=L}+\dot{T} T^{-1} J .
$$

On the other hand, the boundary condition (2.2) implies that $\left.\left(\dot{L} u_{x}+u_{t}\right)\right|_{x=I}=0$. Thus, we can substitute $-\dot{L} u_{x}$ for $u_{t}$ to deduce the formula

$$
\dot{E}=-\left.\dot{L}\left(L^{a} T-L^{b} \dot{L}^{2}\right)\left(u_{x}^{2}\right)\right|_{x=L}+\dot{T} T^{-1} J .
$$


We pause to note that (2.8) agrees, at least qualitatively, with our expectation about the dependence of $E$ on $L$ and $T$. Indeed, $E$ increases as $T$ increases, at constant $L$; and $E$ decreases as $L$ increases, at constant $T$, provided that $\dot{L}^{2}<L^{a-b} T$.

Next, we return to (2.7) and find the derivative of $F$ to be

$$
\dot{F}=\left.\dot{L}\left(x^{b} u^{2}\right)\right|_{x=L}+2 \int_{0}^{L} x^{b} u u_{t} d x,
$$

which reduces to

$$
\dot{F}=2 \int_{0}^{L} x^{h} u u_{t} d x
$$

because $u$ vanishes at $x=L$. A further differentiation gives

$$
\begin{aligned}
\ddot{F} & =\left.2 \dot{L}\left(x^{h} u u_{t}\right)\right|_{x=L}+2 \int_{0}^{L} x^{h}\left(u_{t}^{2}+u u_{t t}\right) d x \\
& =2 K+2 \int_{0}^{L} x^{h} u u_{t t} d x
\end{aligned}
$$

and, on substituting for $x^{b} u_{t t}$ from (2.1), integrating by parts, and appealing to the boundary conditions (including (2.3) if $a=0$ ), we arrive at

$$
\ddot{F}=2 K-2 J \text {. }
$$

Since $E=J+K$, we have, therefore,

$$
J=\frac{1}{2} E-\frac{1}{4} \ddot{F}, \quad K=\frac{1}{2} E+\frac{1}{4} \ddot{F} .
$$

The derivative of $G$ is

$$
\begin{aligned}
\dot{G} & =\left.2 \dot{L}\left(x^{1+h} u_{x} u_{t}\right)\right|_{x=L}+2 \int_{0}^{L} x^{1+h}\left(u_{x t} u_{t}+u_{x} u_{t t}\right) d x \\
& =\left.2 \dot{L}\left(x^{1+h} u_{x} u_{t}\right)\right|_{x=L}+2 \int_{0}^{L}\left(x^{1+h} u_{x t} u_{t}+T x u_{x}\left(x^{a} u_{x}\right)_{x}\right) d x \\
& =\left.2 \dot{L}\left(x^{1+h} u_{x} u_{t}\right)\right|_{x=L}+2 \int_{0}^{L}\left(x^{1+h} u_{x t} u_{t}+a T x^{a} u_{x}^{2}+T x^{1+a} u_{x} u_{x x}\right) d x \\
& =\left.2 \dot{L}\left(x^{1+h} u_{x} u_{t}\right)\right|_{x=L}+2 a J+\int_{0}^{L}\left(T x^{1+a}\left(u_{x}^{2}\right)_{x}+x^{1+h}\left(u_{t}^{2}\right)_{x}\right) d x
\end{aligned}
$$

and, with the help of an integration by parts, we obtain

$$
\dot{G}=\left.\left(2 \dot{L} x^{1+b} u_{x} u_{t}+T x^{1+a} u_{x}^{2}+x^{1+h} u_{t}^{2}\right)\right|_{x=l}-(1-a) J-(1+b) K .
$$

Since, as we know, the boundary condition (2.2) allows us to replace $u_{t}$ by $-\dot{L} u_{x}$ at $x=L$, the derivative

$$
\dot{G}=\left.L\left(L^{a} T-L^{b} \dot{L}^{2}\right)\left(u_{x}^{2}\right)\right|_{x=1}-(1-a) J-(1+b) K
$$

and a glance at (2.8) shows that we have obtained the equation

$$
\dot{E}-\dot{T} T^{-1} J+\dot{L} L^{-1}(\dot{G}+(1-a) J+(1+b) K)=0 .
$$

At this stage, we substitute for $J$ and $K$ from (2.10) to produce the identity

$$
\dot{E}+\frac{1}{2}\left((2+b-a) \dot{L} L^{-1}-\dot{T} T^{-1}\right) E+\frac{1}{4}\left((a+b) \dot{L} L^{-1}+\dot{T} T^{-1}\right) \ddot{F}+\dot{L} L^{-1} \dot{G}=0 \text {. }
$$


If the identity were regarded as a differential equation for $E$, the integrating factor would be

$$
\phi=L^{(2+b-a) / 2} \cdot T^{-1 / 2},
$$

and in terms of the putative adiabatic invariant (2.4), which is

$$
I=\phi E \text {, }
$$

the identity reduces to

$$
\dot{I}+\xi \ddot{F}+\eta \dot{G}=0
$$

where

$$
\xi=\frac{1}{4} \phi\left((a+b) \dot{L} L^{-1}+\dot{T} T^{-1}\right), \quad \eta=\phi \dot{L} L^{-1} .
$$

It is (2.13) which provides the key to the proof that $I$ is an adiabatic invariant; before the proof can be completed, though, certain bounds on $F$. $\dot{F}$, and $G$ are required.

In order to estimate $F$, we begin by remarking that $i$ ce restrictions on $a$ and $b$ ensure the existence of the repeated integral

$$
\int_{0}^{L}\left(\int_{0}^{y} x^{b} d x\right) y^{-a} d y=\frac{1}{1+b} \int_{0}^{L} y^{1+b-a} d y=\frac{L^{2+b-a}}{(1+b)(2+b-a)},
$$

the integrand being nonnegative. Hence, by the Tonelli-Hobson theorem, the repeated integral

$$
\int_{0}^{L}\left(\int_{x}^{L} y^{-a} d y\right) x^{b} d x=\frac{L^{2+b-a}}{(1+b)(2+b-a)} .
$$

According to the boundary condition (2.2),

$$
u(x, t)=-\int_{x}^{L} u_{y}(y, t) d y
$$

and the Schwarz inequality implies that, if $0<x \leqslant L$,

$$
u(x, t)^{2} \leqslant \int_{x}^{L} y^{-a} d y \cdot \int_{x}^{L} y^{a} u_{y}(y, t)^{2} d y \leqslant \int_{x}^{L} y^{-a} d y \cdot T^{-1} J .
$$

On multiplying through by $x^{b}$, integrating with respect to $x$, and using the remark just made, we see that

$$
F \leqslant c^{2} \phi^{2} J
$$

where $c^{2}$ is the reciprocal of $(1+b)(2+b-a)$.

Next, we turn to (2.9) and use the Schwarz inequality and (2.15) to obtain

$$
|\dot{F}| \leqslant 2(F K)^{1 / 2} \leqslant 2 c \phi(J K)^{1 / 2} .
$$

However, the arithmetic-geometric mean inequality yields $2(J K)^{1 / 2} \leqslant J+K=E$, and so

$$
|\dot{F}| \leqslant c I \text {. }
$$

Finally, we rewrite $G$ as

$$
2 T^{-1 / 2} \int_{0}^{l} x^{(2+b-a) / 2} \cdot T^{1 / 2} x^{a / 2} u_{x} \cdot x^{b / 2} u_{t} d x
$$


and appeal to the arithmetic-geometric mean inequality again to deduce the bound

$$
|G| \leqslant T^{-1 / 2} \int_{0}^{l} x^{(2+b-a) / 2}\left(T x^{a} u_{x}^{2}+x^{h} u_{t}^{2}\right) d x .
$$

Since $a<2+b$ it must be that

$$
|G| \leqslant L^{(2+b-a) / 2} \cdot T^{-1 / 2} \cdot \int_{0}^{l}\left(T x^{a} u_{x}^{2}+x^{b} u_{t}^{2}\right) d x,
$$

i.e.,

$$
|G| \leqslant I .
$$

3. Adiabatic invariants for A, B, C; completion of the proof. It is now possible to state what hypotheses are needed on $L$ and $T$ and to complete the proof.

We require $L$ and $T$ to be "slowly-varying" in the sense that (i) there are positive constants $\lambda, \mu, \sigma, \tau$ such that $0<\lambda \leqslant L(t) \leqslant \mu$ and $0<\sigma \leqslant T(t) \leqslant \tau$, (ii) $\dot{L}(t) \rightarrow 0$ and $\dot{T}(t) \rightarrow 0$ as $t \rightarrow \infty$, (iii) the integrals

$$
\int_{0}^{\infty}|\dot{L}|^{2} d t, \quad \int_{0}^{\infty}|\dot{T}|^{2} d t, \quad \int_{0}^{\infty}|\ddot{L}| d t, \quad \int_{0}^{\infty}|\ddot{T}| d t,
$$

converge, and (iv) $\delta<1$, where $\delta$ is defined below, by (3.2).

For the sake of clarity we have stated (ii) separately, even though it is implied by (iii).

The hypotheses have implications for the $\xi$ and $\eta$ which appear in the key identity (2.13). Indeed, we see from (2.14) that $\xi(t) \rightarrow 0$ and $\eta(t) \rightarrow 0$ as $t \rightarrow \infty$ and, therefore, $\xi$ and $\eta$ remain bounded on $0 \leqslant t<\infty$. Furthermore, on calculating the derivatives of $\xi$ and $\eta$, and using (i), we find that $|\dot{\xi}|$ and $|\dot{\eta}|$ do not exceed certain constant multiples of

$$
|\dot{L}|^{2}+|\dot{L} \dot{T}|+|\dot{T}|^{2}+|\ddot{L}|+|\ddot{T}|
$$

and, hence, the integrals

$$
\int_{0}^{\infty}|\dot{\xi}| d t, \quad \int_{0}^{\infty}|\dot{\eta}| d t
$$

converge.

These properties of $\xi$ and $\eta$ ensure that

$$
\delta=\operatorname{Max}_{t \geqslant 0}(c|\xi|+|\eta|)+\int_{0}^{\infty}(c|\dot{\xi}|+|\dot{\eta}|) d t,
$$

which figures in (iv), is finite. This number and the associated

$$
\varepsilon=\frac{\delta}{1-\delta} \exp \left(\frac{\delta}{1-\delta}\right)
$$

measure the slowness with which $L$ and $T$ vary; it scarcely need be pointed out that $L(t)$ and $T(t)$ may undergo large changes on the unbounded interval $0 \leqslant t<\infty$ even when $\delta$ and $\varepsilon$ are small.

The task of showing that $I$ is an adiabatic invariant will have been accomplished if we can show that $I$ is approximately constant in the sense that, when $L$ and $T$ are slowly-varying, $I$ tends to a limit $I(\infty)$ as $t \rightarrow \infty$ and

$$
|I(t)-I(\infty)| \leqslant \varepsilon I(\infty), \quad 0 \leqslant t<\infty .
$$


Let us introduce the sum

$$
H=I+\xi \dot{F}+\eta G
$$

in terms of which the identity (2.13) becomes

$$
\dot{H}=\dot{\xi} \dot{F}+\dot{\eta} G .
$$

According to (2.16) and (2.17), the difference between $H$ and $I$ satisfies

$$
|H-I|=|\xi \dot{F}+\eta G| \leqslant(c|\xi|+|\eta|) I \leqslant \delta I
$$

and, hence, $H \geqslant(1-\delta) I \geqslant 0$ and $I \leqslant(1-\delta)^{-1} H$, provided $\delta<1$. On using (2.16) and (2.17) again, we obtain the estimates

$$
|\dot{F}| \leqslant c(1-\delta)^{-1} H, \quad|G| \leqslant(1-\delta)^{-1} H,
$$

for $\dot{F}$ and $G$. Thus, (3.4) tells us that $H$ satisfies the differential inequality

$$
|\dot{H}| \leqslant(1-\delta)^{-1}(c|\dot{\xi}|+|\dot{\eta}|) H .
$$

The first conclusion to be drawn from (3.7) is that $H$ tends to a limit $H(\infty)$ as $t \rightarrow \infty$. For, a standard argument assures us that either $H$ vanishes identically on $0 \leqslant t<\infty$, in which case $H(\infty)$ exists and equals 0 , or $H$ is strictly positive on $0 \leqslant t<\infty$. In the latter case, the integral

$$
\int_{0}^{\infty}|\overline{\log H}| d t=\int_{0}^{\infty}\left|H^{-1} \dot{H}\right| d t
$$

must converge because the integrals (3.1) converge. It follows that $\log H$ tends to a limit as $t \rightarrow \infty$, and hence $H$ itself tends to a limit.

The convergence of $I$ to a limit now follows almost immediately. Indeed, the argument of the preceding paragraph shows that $H$ is bounded and, by (3.6), so are $\dot{F}$ and $G$. On letting $t \rightarrow \infty$ in (3.4), and recalling that $\xi \rightarrow 0$ and $\eta \rightarrow 0$, we see that $I$ tends to a limit $I(\infty)$, which must coincide with $H(\infty)$.

The next step is to produce explicit bounds on $H, \dot{F}$, and $G$. If $0 \leqslant t \leqslant T<\infty$, the number

$$
|\log H(t)-\log H(T)|=\left|\int_{t}^{T} H^{-1} \dot{H} d t\right|
$$

does not exceed the integral (3.8), which, by virtue of (3.7), does not exceed

$$
(1-\delta)^{-1} \int_{0}^{\infty}(c|\dot{\xi}|+|\dot{\eta}|) d t \leqslant(1-\delta)^{-1} \delta
$$

Hence

$$
H(t) \leqslant \exp \left(\frac{\delta}{1-\delta}\right) H(T) .
$$

On letting $T \rightarrow \infty$ and remembering that $H(\infty)=I(\infty)$, we obtain the estimate

$$
H \leqslant \exp \left(\frac{\delta}{1-\delta}\right) I(\infty)
$$

and, in view of (3.6), the additional estimates

$$
|\dot{F}| \leqslant \frac{c}{1-\delta} \exp \left(\frac{\delta}{1-\delta}\right) I(\infty), \quad|G| \leqslant \frac{1}{1-\delta} \exp \left(\frac{\delta}{1-\delta}\right) I(\infty) .
$$


The last step is to integrate (3.5), thereby obtaining the equation

$$
H(T)-H(t)=\int_{t}^{T}(\dot{\xi} \dot{F}+\dot{\eta} G) d t .
$$

On letting $T \rightarrow \infty$ we see that

$$
I(\infty)-H(t)=\int_{t}^{\infty}(\dot{\xi} \dot{F}+\dot{\eta} G) d t
$$

and, when we substitute for $H$ from (3.4), we find

$$
I(t)-I(\infty)=-\xi(t) \dot{F}(t)-\eta(t) G(t)-\int_{t}^{\infty}(\dot{\xi} \dot{F}+\dot{\eta} G) d t .
$$

Finally, we estimate the right-hand side with the help of (3.9) and arrive at the inequality (3.3); thus the verification that $I$ is an adiabatic invariant is complete.

4. An adiabatic invariant for $D$. Although problem $D$ is not covered by the arguments of Secs. 2 and 3, it can be handled by much the same methods.

To begin with, we note two consequences of the fact that $u=u_{x}=0$ at the moving boundary $x=L$. On differentiating the equation $u=0$ we see that $\dot{L} u_{x}+u_{t}=0$ and, therefore, $u_{t}=0$. On differentiating the equation $u_{x}=0$ we see that $\dot{L} u_{x x}+u_{x t}=0$ and, hence, we have

$$
u=u_{x}=u_{t}=0 \text { and } u_{x t}=-\dot{L} u_{x x} \quad \text { at } x=L .
$$

Next, we express the energy $E$ as the sum of the potential and kinetic energies

$$
J=\int_{0}^{L} u_{x x}^{2} d x, \quad K=\int_{0}^{L} u_{t}^{2} d x,
$$

and introduce the integrals

$$
F=\int_{0}^{L} u^{2} d x, \quad G=2 \int_{0}^{I} x u_{x} u_{t} d x
$$

The derivative of $E$ is

$$
\dot{E}=\left.\dot{L}\left(u_{x x}^{2}+u_{t}^{2}\right)\right|_{x=L}+2 \int_{0}^{L}\left(u_{x x} u_{x x t}+u_{t} u_{t t}\right) d x
$$

and, by virtue of the identity

$$
u_{x x} u_{x x t}+u_{t} u_{t t}=\left(u_{x x} u_{x t}-u_{t} u_{x x x}\right)_{x},
$$

the conditions at $x=0$, and the conditions (4.1), we have

$$
\dot{E}=-\left.\dot{L}\left(u_{x x}^{2}\right)\right|_{x=L} .
$$

This accords, at least qualitatively, with the claim that $L^{2} \cdot E$ is an adiabatic invariant, for it tells us that $E$ decreases as $L$ increases and increases as $L$ decreases.

The first derivative of $F$ is

$$
\dot{F}=2 \int_{0}^{l} u u_{t} d x
$$

and the second derivative is

$$
\ddot{F}=2 K+2 \int_{0}^{L} u u_{t t} d x
$$


The identity

$$
u u_{t t}=\left(u_{x} u_{x x}-u u_{x x x}\right)_{x}-u_{x x}^{2}
$$

and the boundary conditions imply that $\ddot{F}=2 K-2 J$ and, hence,

$$
J=\frac{1}{2} E-\frac{1}{4} \ddot{F}, \quad K=\frac{1}{2} E+\frac{1}{4} \ddot{F},
$$

just as before.

The derivative of $G$ is

$$
\dot{G}=2 \int_{0}^{L}\left(x u_{x t} u_{t}+x u_{x} u_{t t}\right) d x
$$

and, on appealing to the identity

$$
2 x u_{x t} u_{t}+2 x u_{x} u_{t t}=\left(x u_{t}^{2}-2 x u_{x} u_{x x x}+2 u_{x} u_{x x}+x u_{x x}^{2}\right)_{x}-3 u_{x x}^{2}-u_{t}^{2}
$$

and the boundary conditions, we find that

$$
\dot{G}=\left.L\left(u_{x x}^{2}\right)\right|_{x=L}-3 J-K .
$$

Formulae (4.2) and (4.5) imply the identity

$$
\dot{E}+\dot{L} L^{-1}(3 J+K+\dot{G})=0,
$$

and when we substitute for $J$ and $K$ from (4.4) we obtain

$$
\dot{E}+\dot{L} L^{-1}\left(2 E-\frac{1}{2} \ddot{F}+\dot{G}\right)=0 .
$$

If this last were regarded as a differential equation for $E$, the appropriate integrating factor would be $L^{2}$, and, indeed, in terms of $I=L^{2} \cdot E$ it reduces to the identity

$$
\dot{I}+\dot{L} L\left(-\frac{1}{2} \ddot{F}+\dot{G}\right)=0
$$

which plays the same role as did (2.13) in the considerations of Secs. 2 and 3.

As before, certain estimates on $F, \dot{F}$, and $G$ are needed.

Because $u$ vanishes at $x=0$ and at $x=L$ we can write

$$
u(x, t)=-\left(1-\frac{x}{L}\right) \int_{0}^{x} y u_{y y}(y, t) d y-x \int_{x}^{L}\left(1-\frac{y}{L}\right) u_{y y}(y, t) d y .
$$

Hence

$$
|u| \leqslant L\left(\int_{0}^{x}+\int_{x}^{L}\right)\left|u_{y y}\right| d y=L \int_{0}^{L}\left|u_{y y}\right| d y
$$

and, by the Schwarz inequality, $u^{2} \leqslant L^{3} \int_{0}^{L} u_{y y}^{2} d y=L^{3} J$. Thus, on integrating with respect to $x$, we obtain the estimate

$$
F \leqslant L^{4} J
$$

Next, we invoke (4.3), (4.8), and the arithmetic-geometric mean inequality, and find

$$
|\dot{F}| \leqslant 2(F K)^{1 / 2} \leqslant 2\left(L^{4} J K\right)^{1 / 2} \leqslant L^{2}(J+K)=L^{2} E,
$$

i.e.,

$$
|\dot{F}| \leqslant I \text {. }
$$


Finally, we note that, in the light of (4.7),

$$
u_{x}=\int_{0}^{x} \frac{y}{L} u_{y y} d y-\int_{x}^{L}\left(1-\frac{y}{L}\right) u_{y y} d y
$$

and, therefore, $\left|u_{x}\right| \leqslant \int_{0}^{L}\left|u_{y y}\right| d y \leqslant L^{1 / 2} J^{1 / 2}$. Thus,

$$
\int_{0}^{L} u_{x}^{2} d x \leqslant L^{2} J
$$

On returning to the definition of $G$ and using the arithmetic-geometric mean inequality in the form $2\left|u_{x} u_{t}\right| \leqslant L^{-1} u_{x}^{2}+L u_{t}^{2}$ we see that

$$
|G| \leqslant L \int_{0}^{L}\left(L^{-1} u_{x}^{2}+L u_{t}^{2}\right) d x \leqslant L(L J+L K)=L^{2} E
$$

i.e.,

$$
|G| \leqslant I .
$$

It is now possible to prove that $I$ is an adiabatic invariant by introducing the sum $H=I+\dot{L} L\left(-\frac{1}{2} \dot{F}+G\right)$ and arguing, much as before, on the basis of (4.6), (4.9), and (4.10).

Thus, we require $L$ to be "slowly-varying" in the sense that (i) there are positive constants $\lambda$ and $\mu$ such that $0<\lambda \leqslant L(t) \leqslant \mu$, (ii) $\dot{L}(t) \rightarrow 0$ as $t \rightarrow \infty$, (iii) the integrals

$$
\int_{0}^{\infty}|\dot{L}|^{2} d t, \quad \int_{0}^{\infty}|\ddot{L}| d t
$$

converge, and (iv) $\delta<1$, where

$$
\delta=\frac{3}{2} \operatorname{Max}_{t \geqslant 0}|L \dot{L}|+\frac{3}{2} \int_{0}^{\infty}\left(|\dot{L}|^{2}+L|\ddot{L}|\right) d t .
$$

(The factor $3 / 2$ appears because (4.9) and (4.10) imply that $\left|-\frac{1}{2} \dot{F}+G\right| \leqslant \frac{3}{2} I$.)

On setting

$$
\varepsilon=\frac{\delta}{1-\delta} \exp \left(\frac{\delta}{1-\delta}\right)
$$

we can readily verify that, when $L$ is slowly-varying, $I$ tends to a limit $I(\infty)$ as $t \rightarrow \infty$ and

$$
|I(t)-I(\infty)| \leqslant \varepsilon I(\infty), \quad 0 \leqslant t<\infty
$$

\section{REFERENCES}

[1] J. E. Littlewood, Lorentz's pendulum problem, Ann. Physics 21, 232-242 (1963)

[2] J. E. Littlewood, Collected papers, Vol. I, edited by a committee appointed by the London Mathematical Society, Clarendon Press, Oxford, 1982

[3] W. A. Day, Adiabatic invariants for strings and membranes subjected to slowly-larying tension. Quart. Appl. Math. 45, 349-360 (1987) 